\title{
Un système symbolique en construction : l'exemple du bateau birman
}

Maxime Boutry

\section{(2) OpenEdition}

Édition électronique

URL : https://journals.openedition.org/tc/1263

DOI : $10.4000 /$ tc. 1263

ISSN : 1952-420X

Éditeur

Éditions de l'EHESS

\section{Édition imprimée}

Date de publication : 1 décembre 2004

ISSN : 0248-6016

\section{Référence électronique}

Maxime Boutry, "Un système symbolique en construction : l'exemple du bateau birman », Techniques \& Culture [En ligne], 43-44 | 2004, mis en ligne le 22 mars 2006, consulté le 29 septembre 2022. URL: http://journals.openedition.org/tc/1263; DOI : https://doi.org/10.4000/tc.1263

Ce document a été généré automatiquement le 29 septembre 2022.

Tous droits réservés 


\title{
Un système symbolique en construction : l'exemple du bateau birman
}

\author{
Maxime Boutry
}

1 Je saisis l'opportunité de ce numéro consacré aux mythes d'origine des techniques pour développer les recherches sur les constructions techniques et rituelles des bateaux birmans, révélatrices des mécanismes d'appropriation d'un nouvel environnement par une partie de la société birmane. Plutôt que de décrire une adéquation entre un mythe et une technique, il est préférable, en contexte birman (nouveauté des recherches, évolution constante, régionalisme marqué), d'aller en amont et d'expliquer les mécanismes, symboliques, rituels et sociaux qui conditionnent l'apparition d'un mythe. La mythologie et les techniques se construisent en parallèle et j'analyserai ce phénomène en rapport avec le milieu marin. C'est l'occasion de décrire une situation dynamique de création et non de tradition telle qu'elle s'offre généralement au chercheur.

La mer reconsidérée

2 Depuis des siècles, la Birmanie est placée sous le joug d'un pouvoir centralisé qui tire sa force de la richesse de la plaine centrale de l'Irrawaddy, le grenier à riz de la nation. La mer a toujours été un obstacle plutôt qu'une voie de conquête, et dans la culture de ces gens de terre, elle est oubliée, n'apparaissant que tardivement dans la littérature. Les rares mythes qui s'y rapportent entretiennent l'image d'une immensité infranchissable, monde de tous les dangers (Bernot $1995: 85$ ).

3 Avant le développement de la pêche, ces vingt dernières années, l'exploitation des ressources maritimes se limitait à des pratiques individuelles (Robinne 1985). De rares bateaux sillonnaient l'archipel Mergui, alors repaire des pirates. Mais aujourd'hui, souhaitant briser l'isolement séculaire du pays, le gouvernement tente de développer une économie de marché avec l'apport de capitaux étrangers. La pêche se trouve à la pointe de cette nouvelle idéologie libérale. 
4 François Robinne (1994 : 202) parle de retard accumulé; il rapporte que «sur le million de tonnes par an de production potentielle, 565260 tonnes de poissons et crustacés ont été pêchés en 1977-1978». En 2001-2002, la production atteignait déjà les 1300000 tonnes. La division du Tenasserim, dans laquelle se trouve l'archipel Mergui, y participe pour $40 \%$, soit l'équivalent de la production du début des années 1980. Pour réussir à en faire son troisième secteur d'exportation, le gouvernement a mis en place une "économie de marché contrôlée » en privatisant l'ensemble de la filière -fait unique dans l'économie birmane-, ce qui la remet aux mains de compagnies dont les plus importantes achètent, transforment et exportent les meilleurs poissons vers l'ensemble de l'Asie du Sud-Est. Les huit cents îles qui forment l'archipel, étirées sur $400 \mathrm{~km}$ selon un axe nord-sud, sont devenues pour l'État birman un laboratoire économique qui tente d'amener la pêche à un certain seuil de rentabilité, en privilégiant des productions particulières, variant d'une année sur l'autre. Ce contrôle vise également à limiter l'émergence d'une classe moyenne, potentiellement dangereuse.

5 Ce développement concerne une partie des pêcheurs, mais aussi des aventuriers ou réfugiés partis dans les îles éloignées du littoral, dont les seuls occupant étaient jusqu'alors les Moken (nomades marins échappés du monde malais ${ }^{1}$ ).

La naissance d'une tradition

6 Depuis les premiers ouvrages décrivant la flotte de mer birmane, plusieurs changements liés à l'ouverture timide du pays sont survenus; je me limiterai ici à mes propres observations, concernant principalement les bateaux de pêche présents dans l'archipel Mergui, qui sont par ailleurs le point de convergence des techniques et des constructions symboliques associées au milieu marin.

7 La batellerie birmane, nombreuse et d'une grande importance culturelle, de par le rôle des embarcations royales, notamment, et des courses de pirogues rassemblant les foules autour de l'Irrawaddy, a fait l'objet de descriptions précises (Shway Yoe 1963; Ferrars 1996); et les auteurs désireux de répertorier les bateaux croisant au large de la Birmanie s'appuient de manière pertinente sur la tradition fluviale (Ferrars 1996; Charney 1997; Robinne 1994; Gansser et Ivanoff 2000). Cependant, Ivanoff souligne que «la terminologie navale birmane n'est pas assez élaborée pour permettre une classification adéquate des embarcations. C'est pourquoi tous les auteurs ont fait valoir des classifications assez fantaisistes"(2000: 335). Ainsi F. Robinne classe-t-il les embarcations selon leur capacité et leur fonction, alors qu'il semblerait plus judicieux de les distinguer par leur forme. La forme de la poupe, en particulier, permet de recencer quatre grands types de bateaux dans l'archipel Mergui et les villes côtières (Kawthaung au sud et Mergui au nord), qui servent à la petite pêche et plus généralement au déplacement dans les îles (fig. 1 et 2).

8 1) Parlons tout d'abord du lawng hlé: ${ }^{2}$, déjà décrit par Ivanoff (2000), car il apparaît que ce bateau -sans aucun doute le dernier héritier de la tradition fluviale adapté à la navigation en mer- est à l'origine de la classification présentée ici. Outre sa coque monoxyle, c'est bien plus la forme de la poupe -élément le rattachant à la batellerie birmane- qui le distingue des autres bateaux que l'on trouve actuellement dans le sud de la Birmanie (fig. 1). Cette pièce de bois pleine rattachée au monoxyle, incurvée vers le ciel et le plus souvent ornée de décorations sculptées, vaut également à ce bateau le nom de shiema pè' (fig. 2). Elle rappelle la forme des laung-go, utilisés pour le transport fluvial, ou celle des laung-zat de l'Irrawaddy (Ferrars 1996: 132, 135). Encore très 
présent dans l'archipel, ce bateau tend cependant à être modifié ou remplacé par d'autres modèles.

2) Les modifications apportées au traditionnel shiema pè' ont donné naissance au tan: ké: pè' (" pièce dessus poupe ») (fig. 2). Il s'agit d'un inter-médiaire entre les bateaux de pêche semi-artisanale et les chaluts thaïs (walats, wadanes), résultat direct des contraintes économiques et politiques subies par les pêcheurs de l'archipel. C'est, au niveau de la capacité de transport, l'embarcation qui répond le mieux aux besoins en glace et carburant des petits pêcheurs qui n'ont pas les moyens d'investir dans les chaluts détenus par les compagnies de pêche ${ }^{3}$. Le tan: ké: pè' est souvent construit de toutes pièces, mais peut encore dériver d'un shiema pè'.

10 3) Le peshu hlé: («malais bateau»), apparu il y a peu de temps dans les îles, tend lui aussi à supplanter le traditionnel shiema pè' (fig. 1). D'après mes informateurs, il s'agirait d'un modèle rapporté par des Birmans partis quelque temps travailler en Malaisie. Comme souvent en Asie du Sud-Est, les explications avancées pour un changement technique évoquent un héros civilisateur moderne, un voyageur ou un taukay (terme d'origine chinoise signifiant "patron/entrepreneur ») 4 . Il est possible que cette embarcation soit depuis plus longtemps présente sur le littoral, mais son emploi connaît à l'heure actuelle un développement fulgurant. En Birmanie, la nouveauté associée à une volonté de développement aux mains d'un pouvoir centralisé, produit des avancées techniques efficaces et bien acceptées. En effet, le choix de cette embarcation est lié à ses qualités qui en font, parmi les bateaux birmans, l'un des mieux adaptés à la navigation en mer : sa coque profilée et son faible tirant d'eau lui confèrent une plus grande rapidité et la possibilité d'aborder les plages de l'archipel, pour la plupart dépourvues d'infrastructures d'accostage. Ce bateau est également qualifié de u: gyon pè gyon (« tête acérée poupe acérée »), en référence à la poupe bifide originelle en forme de pointe ne subsistant actuellement qu'au niveau de la proue ${ }^{5}$.

11 4) Enfin vient le bhauk tu, entièrement fait de planches assemblées et le plus souvent désigné par le nom de pè pya(t), signifiant «poupe couper» (fig. 1). Ce bateau à la poupe droite est en effet le seul à ne pas posséder la pièce maîtresse qui rejoint les deux bords au niveau de la poupe, appelée pè ya: za taing (fig. 2). Peu usitée, en tout cas pour la pêche, en raison de sa mauvaise tenue en mer, le seul atout de cette embarcation est son faible coût de construction. Sa fonction principale est le transport de marchandises ou de passagers sur de petits trajets. Notons que l'on retrouve les équivalents du u: gyon pè' gyon et du pè' pya $(t)$ à Patani en Thaïlande, respectivement sous les noms de kolè', le bateau traditionnel malais, et du pata kera («cul coupé »), bateau d'origine thaïe (Le Roux \& Cortez 1995). Mais les stratégies adoptées par les pêcheurs des deux endroits diffèrent, car le bateau traditionnel malais est abandonné au profit du «cul coupé » en Thaïlande; c'est l'inverse en Birmanie. On peut y déceler la confirmation de l'importance technique et, comme nous allons le voir également, symbolique de la pièce maîtresse de la poupe en Birmanie.

12 Au regard de la description faite ici, c'est bien la forme de la poupe à laquelle fait référence la totalité des noms de bateaux, qui caractérisent les différents types de constructions, plus que les bordés (Ivanoff 2000) ou la fonction. En plus du monoxyle autrefois commun à la plupart des embarcations fluviales, la poupe du lawng hlé: est le seul caractère hérité de la tradition fluviale. Il est alors logique tout d'abord qu'elle donne au bateau le nom de shiema pè', et qu'avec la diversification des embarcations adaptées de modèles étrangers (thaïs, malais), le terme pè' (poupe) prenne la fonction 
de classificateur. Alors que J. Ivanoff remarquait, à propos de l'origine française du mot bhauk tu, "que les Birmans sont ouverts et qu'ils intègrent dans leur technique des influences extérieures sans complexe et ne se sentent pas obligés de se référer à une tradition unique et invraisemblable» (2000: 335$)$, il s'agit ici du processus inverse, révélateur de la nouveauté et d'une dynamique d'appropriation de l'environnement. Nommer est l'outil indispensable à « des hommes qui pensent et rêvent le monde afin de mieux s'en saisir " (Revel 1990: 43), idée énoncée en référence au monde naturel mais qui convient également à celui des techniques. Cette classification naît dans les îles n'est pas sans reconnaissance pour ses racines continentales et l'héritage de la batellerie. Enfin, nous allons voir qu'elle trouve également une résonance dans la construction symbolique qui accompagne le développement de la pêche, avec le bateau comme principal référent des pêcheurs confrontés à «l'inaccessibilité des horizons marins » (Robinne $1994: 185)$.
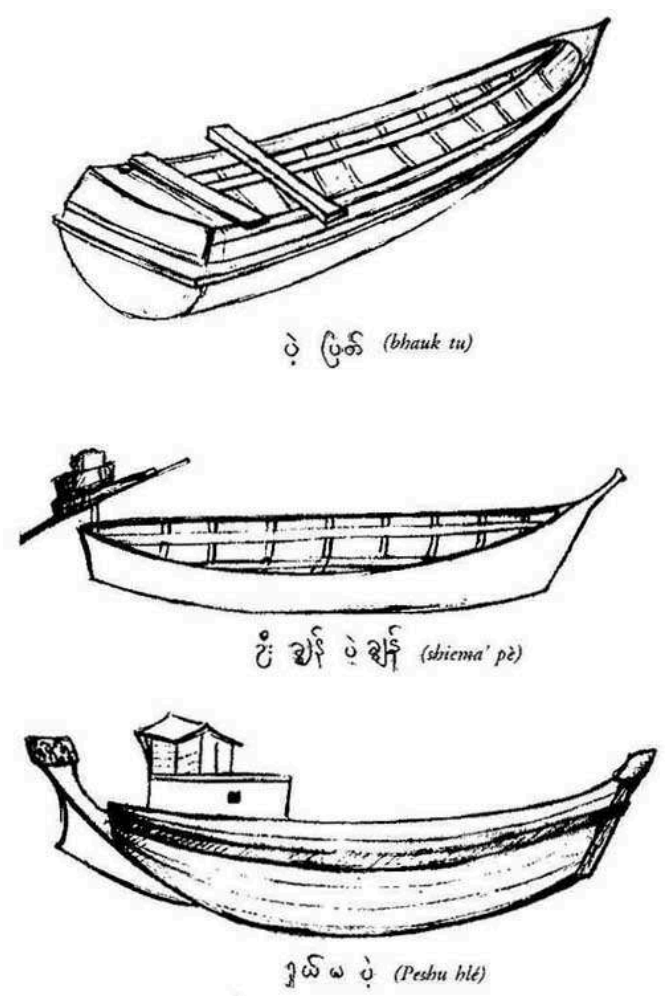
Figure 1. Les principaux bateaux de pêche de l'archipel Mergui

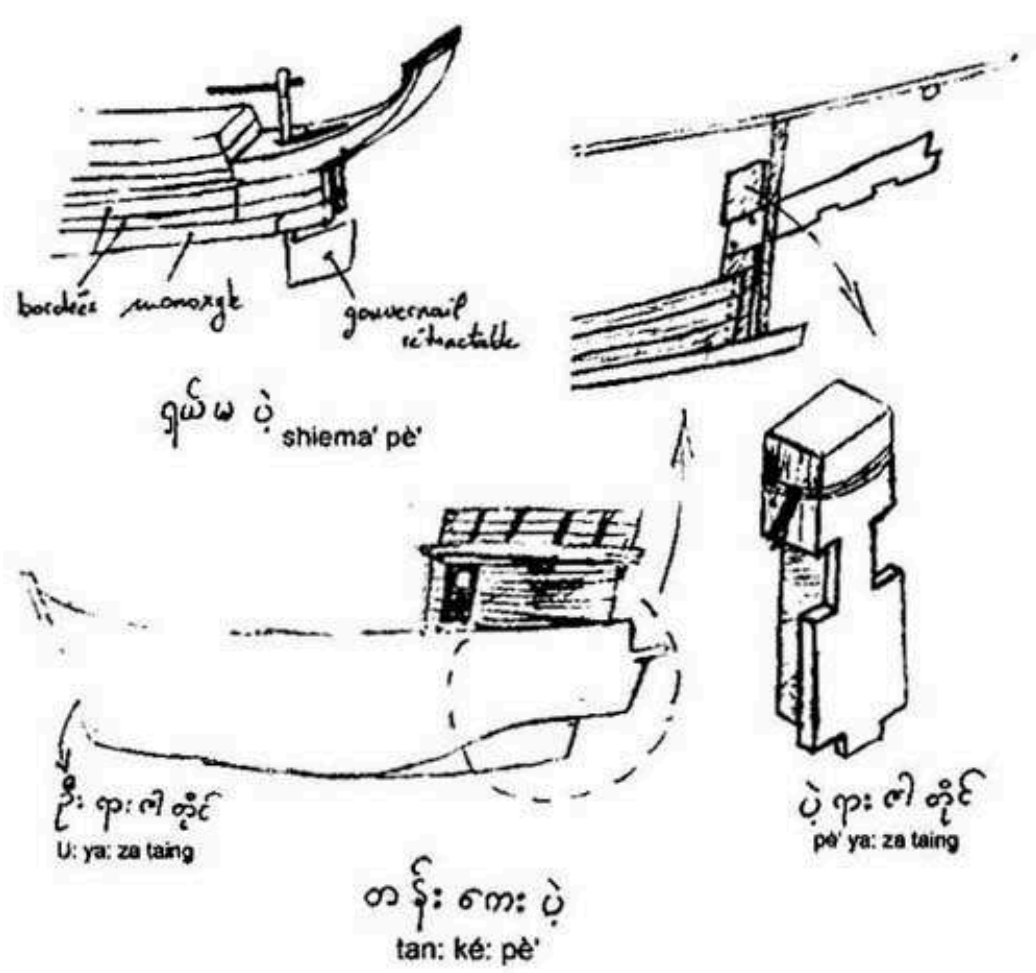

Figure 2. la poupe caractéristique du ', à la base du tan: ké: pè'.

Les constructions symboliques

Dans sa partie « offrandes et rituels ", J. Ivanoff (2000:365) observe déjà que «tous les marins honorent le $n a t^{6}$ situé à la proue du bateau avant chaque départ, et cela quel que soit le bateau ou la forme de sa coque ». Il avance tout d'abord qu'il s'agit de " U Shingi », puis que "certains marins honorent aussi, et parfois surtout, Na Shema [...] situé aussi dans l'étrave ». Il rappelle à juste titre que la liste officielle des $37 n a(t)$ émise par la monarchie birmane, suite à l'impossibilité de faire disparaître le culte animiste antérieur à l'arrivée du bouddhisme, n'est pas exhaustive, puisqu'elle n'inclut pas les nombreux $n a(t)$ locaux et régionaux ${ }^{7}$-dont fait d'ailleurs partie U: Shin Gyi:- et que les $n a(t)$ « se transforment en fonction des besoins et des régions, avatars des nats plus “visibles" ". Les bases du système symbolique ${ }^{8}$ de la pêche en mer et plus généralement de l'appropriation du milieu marin sont ici posées. U: Shin Gyi: («monsieur seigneur grand ») est en effet le na(t) de l'eau salée, reconnu comme tel par tous les pêcheurs interrogés. Même s'il ne fait pas partie du panthéon des $37 \mathrm{na}(\mathrm{t})$, son mythe d'origine est connu à travers toute la Birmanie ${ }^{9}$, et un autel lui est souvent dédié devant les maisons des marins. Cependant, seul le na(t) Ma' Shinma' " possède " le bateau, les pêcheurs, remarquent eux-mêmes que U: Shin Gyi: ne peut être à la fois $n a(t)$ de la mer et $n a(t)$ du bateau.

Le terme " Ma' Shinma' ", signifierait " sœur femme ${ }^{10}$. Cette présence féminine à bord $\mathrm{du}$ bateau se retrouve sous la forme de htanaka, poudre de bois de santal de couleur jaune mélangée à de l'eau, qui forme des motifs abstraits sur la proue, semblables à ceux qui embellissent le visage des Birmanes. Par ailleurs, une boîte contenant tout les attributs d'une femme (robe, collier, boucles d'oreilles, parfum, etc.) est entreposée à la 
base de la proue (elle est exceptionnellement absente lorsqu'elle gêne la manœuvre des engins de pêche). Quoique l'origine de ce $n a(t)$ soit incertaine, au cours de mes enquêtes, plusieurs personnes ( $n a(t) k^{2} a d a w^{11}$ ou intellectuels birmans) ont fait le rapprochement entre Ma' Shinma' et Lemaing, patronne des bateliers et des cultivateurs qui, bien qu'invoquée lors des rituels de possession de $n a(t)$ ne fait pas partie de ces derniers. À ce propos, J. Ivanoff rapporte que Ma' Shinma' est "localement reconnu comme "le nat de l'eau douce" » $(2000: 365)$. Par ailleurs, parmi la variété des mythes que j'ai pu recueillir auprès des marins de l'archipel, certains situent l'origine de l'esprit dans les eaux douces. D'autres, au contraire, la situent sur le littoral ou dans les îles, dans le Tenasserim comme sur la côte arakanaise, beaucoup plus au nord.

Ma' Shinma' vient habiter le bateau au moment de sa construction; elle est en général appelée par le charpentier en charge du chantier, ou par le propriétaire s'il exprime le désir de s'en occuper ${ }^{12}$. Cet appel intervient lors du remplacement des pièces provisoires (a'ngè ya: za taing) par celles qui formeront la proue et la poupe (u: ya: za taing et pè' ya: za taing). L'esprit prend ainsi possession de l'ensemble du bateau en s'installant dans les deux extrémités, là où se rejoignent également les deux bords. La date du rituel est choisie en fonction du calendrier lunaire birman qui définit les jours propices à ce type d'actions et il est effectué avant neuf heures du matin (neuf comme gestation, femme, fécondité). Une fois la proue (u: ya: za taing) fixée, la « tête » est lavée avec de l'« eau d'or » (shwe yé:), de l'eau dans laquelle auront trempé des bijoux ${ }^{13}$. Les offrandes -constituées de riz gluant, de bétel (feuilles et noix d'arec), de feuilles de tabac, de feuilles de thé macérées, de haricots secs et de sésame- sont amenées à la base de la proue. Les bougies sont allumées et l'encens est consumé pendant une prière qui se termine par le nouage d'un bout de tissu bicolore (le plus souvent rouge et blanc), que le propriétaire du bateau remplacera régulièrement ${ }^{14}$. Vient ensuite le tour de la poupe, à laquelle on liera également une pièce de tissu identique. Celle-ci n'est pas changée puisqu'elle disparaît sous le pont et n'est plus accessible une fois la construction terminée. On peut toutefois encore l'observer sur les bateaux hors d'usage dont les planches ont été récupérées pour la construction d'autres navires. Ce cérémonial montre l'importance des deux pièces pour la résidence du na(t), la proue devenant l'objet du culte au quotidien du fait qu'elle est la partie visible, la tête de l'esprit.

Les deux vies de Ma' Shinma'

Malgré le flou autour de son origine, son existence en tant que « $n a(t)$ du bateau » est affirmée par les pêcheurs; elle a sa place parmi les quelques figures mythiques birmanes se rapportant au milieu marin que l'on trouve dans les écrits.

C'est dans le rituel de départ des pêcheurs évoqué par Ivanoff $(2000)^{15}$ que l'on trouve Ma' Shinma', aux côtés notamment de U: Shin Gyi:. Avant une campagne de pêche, le propriétaire doit effectuer un rituel symbolisant le passage du foyer à l'embarcation. Tout d'abord, face à l'autel de U Shin Gyi:-représenté par un tableau où il figure avec sa harpe avec laquelle il arriverait à charmer les animaux les plus cruels, comme le crocodile et le tigre qui l'entourent (Bernot 1995 : 96)-, une prière est dite pour requérir la protection de l'esprit pendant la campagne de pêche. Des encens sont brûlés et transportés sur le bateau en même temps que des offrandes (les mêmes que pour le rituel de construction). Le $n a(t)$ «domestique » est ainsi transporté de la maison au bateau. Puis la proue est lavée et l'on y allume une bougie, et de l'encens est planté en trois endroits : sur le moteur, les hameçons et la proue. C'est Ma' Shinma' qui est cette 
fois-ci sollicitée. L'offrande qui lui est réservée est composée de fleurs et de fruits, dont le nombre et la nature sont laissés au choix du propriétaire. Ils sont souvent au nombre de trois et toujours offerts verts. Enfin, une offrande est faite à Bo Bo Gyi: (terme signifiant " grand-père »), le $n a(t)$ gardien du village. Une noix de coco est passée audessus de la bougie, puis elle est coupée pour en verser l'eau à la mer, de chaque bord du bateau. De la canne à sucre est aussi découpée et jetée à l'eau de chaque côté et devant la proue. Ces opérations consistent à nourrir le $n a(t)$ du village, probablement pour qu'il prenne en charge le foyer des marins pendant leur absence. On remarque dans ce rituel que U: Shin Gyi: opère la transition entre les milieux terrestre et marin, la maison et le bateau. Cela vient probablement de son origine, située d'après le mythe sur une île du delta de l'Irrawaddy, là où mangrove et eau saumâtre font la transition entre les eaux douces et la mer. Mais une fois en mer, seule Ma' Shinma' veille sur le bateau et l'équipage, qui lui consacre une prière quotidienne. On a donc opposition entre les deux esprits, l'un masculin, protecteur des marins et de leur foyer qu'ils emportent à bord, l'autre féminin, symbole de fécondité qui assure le succès de la pêche, et qui pourrait être à l'origine de l'interdiction pour les femmes de monter à bord.

Si le bateau apparaît comme référent d'une identité propre aux pêcheurs, c'est à travers la proue et ses fonctions, au-delà de la vie du bateau stricto sensu, qu'il est possible de le déceler. On trouve parfois des poupes - notamment de lawng hlé:abandonnées sur la plage; elles sont la plupart du temps conservées, ou du moins entreposées dans des endroits où elles ne font pas figure de déchets. Et il est de plus en plus fréquent de les trouver adossées aux maisons ou dans la fourche d'un arbre, façon d'attirer la chance sur le foyer, mais peut-être également de marquer son appartenance à une cer-taine catégorie sociale. En effet, certaines familles de marins se transmettent la proue au long des générations, comme d'autres $n a(t)$ domestiques qui voyagent avec le foyer lors de son exode ${ }^{16}$. Un charpentier du village de La Ngan: -situé dans une île au plein milieu de l'archipel et qui constitue mon terrain principal- possède plusieurs proues appuyées sur la façade de son foyer, légèrement surplombées par un autel à U: Shin Gyi: et par Shin U: Pagotta. C'est dans les îles que la recomposition du monde rituel et symbolique est la plus courante. Dans les milieux considérés comme extrêmes, la création de nouveaux $n a(t)$ est spontanée, car elle répond à un besoin de proximité avec l'environnement que ne peut offrir la religion bouddhiste, trop codifiée. Or les îles représentent sans aucun doute un tel milieu à la manière dont l'exprime le mythe du garudâ qui, parti à la recherche d'une terre au-delà des océan, prit la queue, le corps et la tête d'une baleine pour des îles accueillantes (Cardinaud-Staeyert 1983 : 209-212). Alors pour les pêcheurs, pionniers de cet environnement, Ma' Shinma' est un repère, la femme qu'il ne peuvent avoir en mer, qui garde leur bateau comme elle garderait leur foyer. Dans certaines embarcations, une deuxième extrémité de proue est fixée en deçà de la « vraie " proue. Elle vient d'un ancien bateau appartenant au propriétaire, ou à l'un de ses parents. L'esprit est ainsi respecté, circulant de la sorte d'un navire à l'autre, garante de la sécurité de l'équipage et de la famille. Ma' Shinma' est parfois même représentée à l'intérieur du foyer. En effet, dans la maison d'un popriétaire pêcheur de La Ngan:, la proue d'un de ses anciens bateaux soutient l'autel du $n a(t)$ du foyer, eïn duin $n a(t)$. L'analogie entre ces deux esprits est alors frappante, puisque le dernier est matérialisé par une noix de coco entourée d'un foulard symbolisant sa tête, à l'instar de la proue maquillée de htanaka comme le visage d'une femme ${ }^{17}$.

La révolution symbolique 
19 Ces données suscitent un grand nombre d'hypothèses, mais sont incontestablement révélatrices de l'importance des constructions symboliques dans l'appropriation d'un nouvel environnement, transcendant, en tant que référent d'une communauté, les essais techniques dus à l'essor de la pêche. On voit se nouer la révolution symbolique grâce à un na(t) adaptable, autour duquel une partie de la société birmane réinvente un monde surnaturel à sa mesure.

20 Ainsi, nous avons vu que la classification des bateaux s'établit en référence à la forme de la poupe, dernier élément caractéristique de l'identité birmane car hérité de la tradition fluviale. Parallèlement, si Ma' Shinma', dont les origines sont incertaines mais laissent supposer une affiliation aux eaux douces, on voit là que les évolutions techniques et rituelles sont simultanées. Mais alors que les premières sont maintenues par un système économique et politique très contraignant, les secondes n'ont pas de limites et tendent vers la construction d'un système symbolique de plus en plus homogène. Et à ce titre, au fur et à mesure que la transition entre eaux douces et eaux salées s'effectue, Ma' Shinma' s'éloigne indubitablement de ses origines pour devenir l'élément central d'un système symbolique propre au milieu marin et insulaire. De même, nous avons vu que lors du rituel de départ des pêcheurs, le $n a(t)$ U: Shin Gyi: était transporté du foyer au bateau. La dualité homme/femme entre l'esprit de la mer et celui du bateau fait également apparaître l'opposition entre "domestiqué » et "sauvage». En effet, l'«Esprit des eaux salées» est connu depuis longtemps, "domestiqué", et fut transporté dans le sud de la Birmanie depuis le delta de l'Irrawaddy, accompagnant le foyer. Or il est également présent sur le bateau, puisqu'il y est transporté lors du rituel; et sur la plupart des cargos et des petits bateaux de pêche possédant un habitacle, il y a un tableau où il figure, semblable à celui que l'on retrouve dans les autels près des maisons. Ma' Shinma' appartient au domaine du "sauvage " et U Shin Gyi: au monde "domestiqué ». Le bateau est livré à un esprit féminin, certes plus propice à la réussite car symbole de fécondité, mais un esprit aux origines incertaines, compagne des pêcheurs, eux-mêmes au bas de l'échelle sociale birmane. Et cependant, elle opère au fur et à mesure une domestication de l'environnement. La proue est maintenant ramenée à terre, parfois même dans les foyers, et tout porte à croire qu'il s'agit de Ma' Shinma' que l'on tente d'intégrer au panthéon. Mais cet esprit reste mal défini, et c'est pour cela que les Birmans de l'archipel rechignent à en parler. Ses pouvoirs sont mal connus et pas vraiment arrêtés. Ma' Shinma' recouvre un monde surnaturel inachevé, que les pêcheurs redoutent de nommer car ils n'en connaissent pas encore toutes les fonctions.

21 Ce n'est qu'avec la diversification très récente des embarcations de pêche, à la source du processus de colonisation des îles éloignées du continent, que l'on peut dégager une structure dans l'appréhension des constructions navales, où les choix techniques répondent au contexte économique strict tel qu'il est imposé aux petits pêcheurs, et autour desquels se construit un système symbolique indispensable à l'appropriation du milieu marin par ces "aventuriers». Et l'apparition, ou la reconnaissance de Ma' Shinma' par l'ensemble des pêcheurs comme esprit du bateau transforme celui-ci -et particulièrement la proue- en objet de référence pour une partie de la société birmane. Par ailleurs, les histoires autour de ce $n a(t)$, hétérogènes, fournissent pourtant de nombreux éléments qui laissent présager le développement d'une mythologie pour l'instant absente de la littérature, mais qui pourrait bien rattraper un jour le vide laissé par le milieu marin dans la culture birmane ${ }^{18}$. 
innovations techniques, pour fédérer une communauté confrontée à ce nouvel environnement. Et c'est en toute logique le bateau qui en est le centre, support principal de l'appropriation du milieu marin et insulaire pour les Birmans de l'archipel Mergui devenu laboratoire social et culturel.

\section{BIBLIOGRAPHIE}

Bernot, Denise

1995. «Les mythes de l'eau et de l'océan en Birmanie », pp. 85-89, in Les Mythes de l'eau et de l'océan en Orient, Océanis. Paris : Institut Océanographique.

Brac de La Perrière, Bénédicte

1989. Les Rituels de possession en Birmanie : du culte d'État aux cérémonies privées. Paris :

Recherche sur les Civilisations.

Boutry, Maxime

2003. La Ngan, entre oubli et liberté. Mémoire de DEA Aménagement Développement, Environnement. Orléans : Université des Lettres, Langues et Sciences humaines.

Cardinaud-Stayaert, Marie-Hélène

1983. Le « Conte de Pourquoi » en Birmanie, traduction et étude critique d'un corpus de contes étiologiques. Université Paris III (thèse de doctorat de 3ème cycle, multigr.).

Charney, Michael

1997. "Shallow-draft boats, guns, and the Aye-ra-wa-ti : continuity and change in ship structure and river warfare in Precolonial Mynanmar », Oriens Extremus 40, $1: 16-23$.

Ferrars, Max et Bertha

1996. Burma. Bangkok : Ava Publishing House.

Gansser, Luca \& Jacques Ivanoff

2000. « Aperçus d'une évolution technique et rituelle en Basse-Birmanie : de la batellerie à la pêche litorale et inter-îlienne », Techniques \& culture 35-36 : 329-372.

Ivanoff, Jacques

" Manger ou ne pas manger du riz ? Le choix moken (archipel des Mergui) », Techniques \& Culture 31-32: 331-346.

Ivanoff, Jacques \& Thierry Lejard

2001. Mergui et les Limbes de l'Archipel Oublié: impressions, observations et description de quelques îles au large du Tenasserim. Paris : Ketos/ Bangkok : White Lotus press (en collaboration avec L. et G. Gansser).

Revel, Nicole

1990. Fleurs de paroles, Histoire Naturelle Palawan, tome I : Les Dons de Nägsalad. Peeters-

France/SELAF («Ethnosciences »).

Robinne, François

1985. « Le littoral oublié des Birmans », Asie du Sud-Est et Monde Insulindien XIV (3-4) : 181-184. 
1994. «Pays de mer et gens de terre. Logique sociale de la sous-exploitation du domaine maritime en Asie du Sud-Est continentale », Bulletin de l'École Française d'Extrême Orient 81 : 181-216.

Rodrigues, Yves

Nat-Pwe: Burma's Supernatural Sub-Culture. Gartmore : Kiscadale Publications.

Temple, Richard Carnac

1991. The Thirty-seven Nats : a Phase of Spirit Worship Prevailing in Burma, 1906. Londres : W. Criggs.

\section{NOTES}

1. À ce sujet, voir les travaux de Jacques Ivanoff (Ivanoff \& Lejard 2001).

2. À propos de la transcription des noms birmans : à l'exception du vocabulaire apparaissant dans les citations dont l'orthographe a été respectée, j'utilise une transcription phonologique, qui se veut la plus proche possible de l'écriture, notamment des trois tons de la langue. Le 1er (montant), le 2ème (descendant) et le 3ème (montant-descendant) sont respectivement formalisés par _', _, _.: Les arrêts glottaux sont marqués par des parenthèses entourant la dernière consonne. Ainsi le mot « esprit », s'écrira $n a(t)$, terminé par un arrêt glottal sur le son « $t$ ». Le « $h$ » avant une consonne signifie qu'elle est aspirée. En revanche, th est un peu l'équivalent du son employé pour this en anglais. Le « $\mathrm{ch}$ » français est traduit par sh (différent de hs, «s » aspiré). Enfin, la combinaison en birman des consonnes « $k$ » et « $y$ » donnent les « dj » ou «tch», ici retranscris par gy. Exemple : grand = gyi: (prononcer « djii »).

3. Par ailleurs, ce sont elles qui fixent les prix du poisson. Ainsi, malgré la privatisation de la filière, le gouvernement qui taxe directement ces compagnies dont le plus gros de la production est destiné à l'export et qui garde en dépit des apparences un fort contrôle sur leur fonctionnement, n'a pas à craindre l'émergence d'une classe de pêcheurs plus riche et donc moins contrôlable

4. Citons par exemple le héros Gaman, qui apporta aux Moken le riz, remplaçant l'igname, en même temps qu'il engendra leur exode (Ivanoff 1998)

5. La poupe a probablement perdu sa forme de départ pour permettre le positionnement axial du moteur.

6. En birman, le terme $n a(t)$ désigne trois types d'esprits : les divinités de la mythologie hindoue incorporées aux croyances birmanes, les esprits non personnifiés de la nature et les esprit birmans (Brac de la Perrière 1989). Il est fait référence ici à cette dernière catégorie.

7. On peut cependant considérer qu'un $n a(t)$ fait partie du panthéon officiel lorsqu'il fait l'objet de possession par les na(t) kadaw (" épouse de $n a(t)$ », terme désignant les médiums spécialistes du culte des na(t)), ce qui est par exemple le cas de la « divinité bufflesse » qui ne fait pourtant pas partie de la liste des 37 (B. Brac de la Perrière, comm. pers.). Pour plus de précisions sur le culte des na(t), voir B. Brac de la Perrière (1989).

8. Les hommes s'appuient entre autres sur le monde surnaturel pour s'approprier leur environnement. Ce sont les relations entre ces trois éléments (monde surnaturel/ homme/environnement) qui forment ce que j'entends ici par « système symbolique ». 9. Voir à ce propos Rodrigues (1992 : 47) et Boutry (2003).

10. «Ma » provenant de ae'ma: « sœur » est un terme d'adresse employé à l'intention d'une femme d'âge sensiblement égal à celui de l'interlocuteur. Quant à « shinma' », il 
s'agit d'un terme employé par les maris à l'adresse de leur femme (Myanmar-English Dictionnary...).

11. Signifie littéralement « épouse de $n a(t)$ ». Terme désignant les médiums spécialistes du culte des $n a(t)$, et plus largement toute personne pouvant être possédée au moins une fois par un esprit (Brac de la Perrière 1989 : 52).

12. Je n'ai pu assister à ce rituel mais il m'a été décrit par deux charpentiers de Mergui et du village de La Ngann (au centre de l'archipel Mergui).

13. Les bijoux peuvent rappeler le caractère féminin de l'esprit. À noter également que porter de l'or est synonyme de richesse et donc de réussite sociale, ce qui a probablement pour rôle d'attirer la bonne fortune sur le bateau.

14. Il faut noter que les anciens bouts de tissus sont rarement jetés et qu'ils sont de préférence liés à une autre pièce du bateau, comme le mât ou parfois les perches servant à ramener les filets de la pêche au lamparo, pour « attirer les prises ». 15. L'intervention, entre autres, de ces deux $n a(t)$ dans le rituel de départ est probablement à l'origine de la confusion entre U: Shin Gyi: et Ma' Shinma' faite par l'auteur.

16. Donnons l'exemple de la divinité bufflesse, à laquelle les émigrés de la région de Pegu (ancienne capitale mône), où a lieu l'histoire du na(t), dédient un autel situé devant la façade de leur maison, rappelant ainsi leur origine.

17. Ce rapprochement est d'autant plus intéressant que la plupart des $n a(t)$ apparaissent représentés dans des tableaux ou sous forme de statuettes (pour les représentations des $n a(t)$, voir notamment R.-C. Temples 1991).

18. Il n'est pas question de développer ici les différents mythes de Ma' Shinma', car cela ferait l'objet d'un article à part entière, bien que mes recherches ne me permettent pas encore de présenter une analyse satisfaisante. Notons cependant que plusieurs éléments, l'« esprit poisson » ou encore le "pinlèson », cités par les marins comme la matérialisation dans le monde des vivants du $n a(t)$, laissent supposer qu'il existe une certaine tradition orale liée au milieu marin, peu connue, mais bien réelle. Les modes de transmission du savoir - rituels, traditions orales, etc. - font partie de mes recherches actuelles.

\section{RÉSUMÉS}

La mer, traditionnellement ignorée de la culture birmane tournée vers la riziculture et les eaux douces, pénètre les sphères des pratiques rituelles des habitants de l'archipel Mergui. Le développement rapide et récent de la pêche impose de nouveaux rapports socio-économiques. À la lumière des données recueillies pendant six mois de terrain, il convient d'apporter des précisions aux recherches préliminaires de Jacques Ivanoff. En effet, l'identité et le rôle technique et social du bateau comme des mythes afférents restent à étudier. L'auteur présente ici des hypothèses sur un système symbolique en construction s'articulant sur les relations entre l'homme, l'environnement, les mythes et plus globalement le monde surnaturel, propres aux pêcheurs birmans de l'archipel Mergui. Le bateau et le $n a(t)$ (esprit) qui y réside sont ici 
considérés comme l'élément essentiel de ce système et comme le support principal de l'appropriation du milieu marin et insulaire.

A symbolic system under construction. The example of Burmese ships

Traditionaly, Burmese do not have got a sea culture, they were involved in rice growing and fresh waters. But the new and recent development of fishing have lead them to new socio-economic relations. After six months fieldwork, J. Ivanoff previous'work can be completed. The identity, the social and technical role of the boat and related myths need to be studied. I present here an hypothesis on a symbolic system under construction which is linked, in the Mergui Archipelago, to man, environment, myths and more generally to supernatural world. The boat, and the nat(s) (spirit) living in it, are considered as the main elements of this system and the main means for the appropriation of the marine and insulary environment.

\section{Un sistema simbólico en construcción : el ejemplo del barco birmano}

Ignorado tradicionalmente por la cultura birmana que se orientaba hacia el cultivo de arroz y las aguas dulces, el mar va penetrando las esferas de las prácticas rituales en el archipiélago Mergui. El desarrollo tan reciente como rápido de la pesca acarrea nuevas relaciones socio-económicas. A la vista de los datos recogidos durante seis meses de trabajo de terreno, es conveniente proporcionar ciertas precisiones para completar las investigaciones preliminares de Jacques Ivanoff. Efectivamente, la identidad y el papel técnico y social del barco, así como los mitos que les corresponden, quedan por estudiar. El autor presenta unas hipótesis respecto a un sistema simbólico en construcción que se articula con las relaciones entre el hombre, el medio natural, los mitos y, globalmente, el mundo sobrenatural - un sistema propio de los pescadores birmanos del archipiélago Mergui. El barco y su nat(t) (especie de genio) son los elementos esenciales de este sistema. Son también el apoyo principal de la apropiación del medio marítimo e insular.

\section{INDEX}

Mots-clés : bateau, pêche, mer, Birmanie, Archipel Mergui, na(t), symbolique

Keywords : boat, fishing, sea, Burma, Mergui Archipelago, symbols

\section{AUTEUR}

\section{MAXIME BOUTRY}

Techniques et culture, 27 rue Paul Bert, 94204 Ivry Cedex. Université des Lettres, Langues et Sciences sociales, Orléans 